\title{
Provider Perceptions of Opioid Safety Measures in VHA Emergency Departments and Urgent Care Centers
}

\author{
Nathalie Dieujuste; Rachel Johnson-Koenke, LCSW, PhD; Manuel Celedon, MD; Zahir Basrai, MD; \\ Melissa Christopher, PharmD; Jason Smith, PharmD; and Comilla Sasson, MD
}

Background: A priority for Veterans Health Administration (VHA) leadership is increasing access to lifesaving treatment, particularly naloxone distribution and medication-assisted treatment (MAT) for opioid use disorder (OUD) for veterans. To date, these practices are not widely done in the VHA emergency departments (ED) and urgent care centers (UCC).

Methods: The goal of this research was to understand advanced care provider perceptions of barriers and facilitators to naloxone distribution or MAT initiation in VHA ED/UCCs. We developed and disseminated a survey to VHA ED and UCC advanced care providers, including medical doctors (MD/DO), physician assistants (PAs), and nurse practitioners (NPs). Descriptive statistical analysis was conducted.

Results: There was $16.7 \%$ response rate (372 out of 2228 providers) from 103 of 132 sites across all VA regions. The top barrier for ED/UCCs providers to both naloxone and
MAT initiation was the feeling that it was beyond their scope of practice $(35.2 \%$ and $53.2 \%$, respectively). Other reported barriers to MAT initiation included unclear follow-up plan and system for referral of care $(50.1 \%)$ and feeling uncomfortable using MAT medications (28.8\%). Top facilitators for prescribing naloxone included pharmacist who could help prescribe/educate the patient on the medication $(44.6 \%)$ and patient knowledge of medication options to help overdose $(31.7 \%)$. The top facilitator for MAT initiation from the ED/UCC was additional VA-based same day treatment options (34.9\%).

Conclusions: Present findings offer a look into possible challenges to address or opportunities to leverage when considering or developing an ED/UCC-based naloxone distribution or MAT-initiation implementation program in VHA facilities.
Author affiliations can be found at the end of the article.

Correspondence: Nathalie Dieujuste (nathalie.dieujuste@va.gov)

Fed Pract. 2021;38(9). Published online September 13. doi:10.12788/fp.0179
T he United States is facing an opioid crisis in which approximately 10 million people have misused opioids in the past year, and an estimated 2 million people have an opioid use disorder (OUD). ${ }^{1} \mathrm{Com}-$ pared with the general population, veterans treated in the Veterans Health Administration (VHA) facilities are at nearly twice the risk for accidental opioid overdose. ${ }^{2}$ The implementation of opioid safety measures in VHA facilities across all care settings is a priority in addressing this public health crisis. Hence, VHA leadership is working to minimize veteran risk of fatal opioid overdoses and to increase veteran access to medication-assisted treatments (MAT) for OUD. ${ }^{3}$

Since the administration of our survey, the VHA has shifted to using the term medication for opioid use disorder (MOUD) instead of MAT for OUD. However, for consistency with the survey we distributed, we use MAT in this analysis.

Acute care settings represent an opportunity to offer appropriate opioid care and treatment options to patients at risk for OUD or opioid-related overdose. VHA facilities offer 2 outpatient acute care settings for emergent ambulatory care: emergency departments (EDs) and urgent care centers (UCCs). Annually, these settings see an estimated 2.5 million patients each year, making EDs and UCCs critical access points of OUD care for veterans. Partnering with key national VHA stakeholders from Pharmacy Benefits Management (PBM), the Office of Emergency Medicine, and Academic Detailing Services (ADS), we developed the Emergency Department Opioid Safety Initiative (ED OSI) aimed at implementing and evaluating opioid safety measures in VHA outpatient acute care settings.

The US Department of Veterans Affairs (VA)/Department of Defense (DoD) Clinical Practice Guidelines for Opioid Therapy for Chronic Pain (CPG) makes recommendations for the initiation and continuation of opioids, risk mitigation, taper of opioids, and opioid therapy for acute pain in VHA facilities. ${ }^{4}$ Using these recommendations, we developed the broad aims of the ED OSI quality improvement (QI) program. The CPG is clear about the prioritization of safe opioid prescribing practices. New opioid prescriptions written in the ED have been associated with continued and chronic opioid use..$^{5}$ At the time of prescription, 
patients not currently and chronically on opioids who receive more than a 3-day supply are at increased risk of becoming long-term opioid users. ${ }^{6}$ Given the annual volume of patients seen, VHA ED/UCCs are a crucial area for implementing better opioid prescribing practices.

The CPG also includes recommendations for the prescribing or coprescribing of naloxone rescue kits. The administration of naloxone following opioid overdose has been found to be an effective measure against fatal overdose. Increasing provider awareness of common risk factors for opioid-related overdose (eg, frequent ED visits or hospitalizations) helps facilitate a discussion on naloxone prescribing at discharge. Prior studies provide evidence that naloxone distribution and accompanying education also are effective in reducing opioid overdose mortality and ED visits related to adverse opioidrelated events. ${ }^{7,8}$

Similarly, the guidelines provide recommendations for the use of MAT for veterans with OUD. MAT for OUD is considered a first-line treatment option for patients with moderate-to-severe OUD. When used to treat patients with unsafe opioid use, this treatment helps alleviate symptoms of withdrawal, which can increase opioid taper adherence and has a protective effect against opioid overdose mortality. ${ }^{9}$ MAT initiated in the ED can increase patient engagement to addiction services. ${ }^{10}$

These 3 CPG recommendations serve as the basis for the broad goals of the ED OSI program. We aim to develop, implement, and evaluate programs and initiatives to (aim 1) reduce inappropriate opioid prescribing from VHA EDs; (aim 2) increase naloxone distribution from VHA EDs; and (aim 3) increase access to MAT initiation from VHA EDs through the implementation of ED-based MAT-initiation programs with EDs across the VHA. Aim 1 was a focused and strategic QI effort to implement an ED-based program to reduce inappropriate opioid prescribing. The ED OSI prescribing program offered a 4-step bundled approach: (1) sharing of opioid prescribing dashboard data with ED medical director and academic detailer; (2) education of ED providers and implementation of toolkit resources; (3) academic detailers conduct
TABLE 1 Respondent Characteristics

\begin{tabular}{lc} 
Characteristics & $\begin{array}{c}\text { Respondents, No. (\%) } \\
(\mathbf{N}=372)\end{array}$ \\
\hline Provider type & $281(75.5)$ \\
Medical doctor & $61(16.4)$ \\
Nurse practitioner & $30(8.1)$ \\
Physician assistant & \\
\hline Time out of school & $25(6.72)$ \\
$0-5$ y & $48(12.90)$ \\
6-10y & $299(80.38)$ \\
$>$ 10 y & \\
\hline Facility type & $301(80.91)$ \\
ED & $71(19.09)$ \\
UCC & \\
\hline Site complexitya & $144(38.71)$ \\
1a ED & $53(14.25)$ \\
1b ED & $60(16.13)$ \\
1c ED & $12(3.23)$ \\
1a UCC & $10(2.69)$ \\
1b UCC & $6(1.61)$ \\
1c UCC & $87(23.39)$ \\
Levels 2 and 3 &
\end{tabular}

Abbreviations: ED, emergency department; UCC, urgent care center. aSite complexity levels: level 1 (a, b, c), high patient volume and patient risk, high levels of teaching/research; level 2, medium patient volume and risk, medium levels of teaching/research; level 3 , low levels of patient complexity, low patient volume, little or no teaching/research.

audit and feedback session(s) with highest prescribers; and (4) quarterly reports of opioid prescribing data to ED providers.

Results from the pilot suggested that our program was associated with accelerating the rate at which ED prescribing rates decreased. ${ }^{11}$ In addition, the pilot found that ED-based QI initiatives in VHA facilities are a feasible practice. As we work to develop and implement the next 2 phases of the QI program, a major consideration is to identify facilitators and address any existing barriers to the implementation of naloxone distribution (aim 2) and MATinitiation (aim 3) programs for treatmentnaive patients from VHA EDs. To date, there have been no recent published studies examining the barriers and facilitators to use or implementation of MAT initiation or naloxone distribution in VHA facilities or, more specifically, from VHA EDs. ${ }^{12}$ As part of our QI program, we set out to better understand VHA ED provider perceptions of barriers and facilitators to implementation of programs aimed at increasing naloxone distribution and initiation of MAT for treatment-naive patients in the ED. 
TABLE 2 Health Care Provider Barriers to Naloxone Distribution

\begin{tabular}{lcccc} 
& \multicolumn{4}{c}{ Health Care Providers, No. (\%) } \\
\cline { 2 - 5 } Barriers & Physicians & $\begin{array}{c}\text { Nurse } \\
\text { Practitioners }\end{array}$ & $\begin{array}{c}\text { Physician } \\
\text { Assistants }\end{array}$ & All \\
\hline Facility leadership not supportive & $15(5.3)$ & $2(3.3)$ & $2(6.7)$ & $19(5.1)$ \\
\hline Not necessary & $21(7.5)$ & $3(4.9)$ & $3(10.0)$ & $27(7.3)$ \\
\hline I am uncomfortable prescribing naloxone & $32(11.4)$ & $9(14.8)$ & $4(13.3)$ & $45(12.4)$ \\
\hline Other & $32(11.4)$ & $4(6.6)$ & $1(3.3)$ & $46(12.6)$ \\
\hline Cost & $42(14.9)$ & $6(9.8)$ & $2(6.7)$ & $50(13.4)$ \\
\hline $\begin{array}{l}\text { Veteran inability to use naloxone } \\
\text { Required time to prescribe naloxone }\end{array}$ & $62(22.1)$ & $18(29.5)$ & $5(16.7)$ & $85(22.8)$ \\
\hline $\begin{array}{l}\text { Beyond the scope of emergency } \\
\text { department or urgent care clinic providers }\end{array}$ & $91(32.4)$ & $17(27.9)$ & $14(46.7)$ & $122(32.8)$ \\
\hline $\begin{array}{l}\text { Veterans perceived stigma that naloxone is } \\
\text { used only for opioid abuse or misuse }\end{array}$ & $96(34.2)$ & $19(31.1)$ & $9(30.0)$ & $124(33.3)$ \\
\hline
\end{tabular}

\section{METHODS}

This project received a QI designation from the Office of PBM Academic Detailing Service Institutional Review Board at the Edward Hines, Jr. Veterans Affairs Hospital VA Medical Center (VAMC). This designation was reviewed and approved by the Rocky Mountain Regional VAMC Research and Development service. In addition, we received national union approval to disseminate this survey nationally across all VA Integrated Service Networks (VISNs).

\section{Survey}

We worked with VHA subject matter experts, key stakeholders, and the VA Collaborative Evaluation Center (VACE) to develop the survey. Subject matter experts and stakeholders included VHA emergency medicine leadership, ADS leadership, and mental health and substance treatment providers. VACE is an interdisciplinary group of mixed-method researchers. The survey questions aimed to capture perceptions and experiences regarding naloxone distribution and new MAT initiation of VHA ED/UCC providers.

We used a variety of survey question formats. Close-ended questions with a predefined list of answer options were used to capture discrete domains, such as demographic information, comfort level, and experience level. To capture health care provider (HCP) perceptions on barriers and facilitators, we used multiple-answer multiple-choice questions. Built into this question format was a free-response option, which allowed respondents to offer additional barriers or facilitators. Respondents also had the option of not answering individual questions.

We identified physicians, nurse practitioners (NPs), and physician assistants (PAs) who saw at least 100 patients in the ED or UCC in at least one 3-month period in the prior year and obtained an email address for each. In total, 2228 ED or UCC providers across 132 facilities were emailed a survey; $1883(84.5 \%)$ were ED providers and 345 (15.5\%) were UCC providers.

We used Research Electronic Data Capture (REDCap) software to build and disseminate the survey via email. Surveys were initially disseminated in late January 2019. During the 3-month survey period, recipients received 3 automated email reminders from REDCap to complete the survey. Survey data were exported from REDCap. Results were analyzed using descriptive statistics analyses with Microsoft Excel.

\section{RESULTS}

One respondent received the survey in error and was excluded from the 
TABLE 3 Facilitators to Naloxone Distribution From ED and UCC

\begin{tabular}{|c|c|c|c|c|}
\hline \multirow[b]{2}{*}{ Facilitators } & \multicolumn{4}{|c|}{ Health Care Providers, No. (\%) } \\
\hline & Physicians & $\begin{array}{c}\text { Nurse } \\
\text { Practitioners }\end{array}$ & $\begin{array}{l}\text { Physician } \\
\text { Assistants }\end{array}$ & All \\
\hline Other & $23(8.2)$ & $3(4.9)$ & $3(10.0)$ & $29(7.8)$ \\
\hline $\begin{array}{l}\text { Being able to spend more time to educate } \\
\text { patient about medication }\end{array}$ & $34(12.1)$ & $3(4.9)$ & $3(10.0)$ & $40(10.8)$ \\
\hline $\begin{array}{l}\text { Videos and/or handouts for patients with } \\
\text { education about medication }\end{array}$ & $57(20.3)$ & $12(19.7)$ & $6(20.0)$ & $75(20.2)$ \\
\hline $\begin{array}{l}\text { Automated prompt in CPRS for those patients } \\
\text { who are being prescribed opioids }\end{array}$ & $57(20.3)$ & $18(29.5)$ & $3(10.0)$ & $78(21.0)$ \\
\hline Patient desire to use opioids safely & $75(26.7)$ & $16(26.2)$ & $8(26.7)$ & $99(26.6)$ \\
\hline Facility leadership interest in opioid safety & $79(28.1)$ & $15(24.6)$ & $6(20.0)$ & $100(26.9)$ \\
\hline $\begin{array}{l}\text { Societal attitudes such as a shift away from } \\
\text { using opioids to manage pain }\end{array}$ & $87(31.0)$ & $13(21.3)$ & $4(13.3)$ & $104(28.0)$ \\
\hline $\begin{array}{l}\text { Patient knowledge of medication options to help } \\
\text { overdose }\end{array}$ & $92(32.7)$ & $20(32.8)$ & $6(20.0 \%)$ & $118(31.7)$ \\
\hline $\begin{array}{l}\text { Pharmacist who could help prescribe and help } \\
\text { educate the patient on the medication }\end{array}$ & $126(44.8)$ & $28(45.9)$ & $12(40.0)$ & $166(44.6)$ \\
\hline
\end{tabular}

analysis. The survey response rate was 16.7\%: 372 responses from 103 unique facilities. Each VISN had a mean 20 respondents. The majority of respondents $(\mathrm{n}=$ 286, 76.9\%) worked in highly complex level 1 facilities characterized by high patient volume and more high-risk patients and were teaching and research facilities. Respondents were asked to describe their most recent ED or UCC role. While 281 respondents $(75.5 \%)$ were medical doctors, 61 respondents (16.4\%) were NPs, 30 (8.1\%) were PAs, and 26 (7.0\%) were ED/ UCC chiefs or medical directors (Table 1). Most respondents (80.4\%) reported at least 10 years of health care experience.

The majority of respondents (72.9\%) believed that HCPs at their VHA facility should be prescribing naloxone. When asked to specify which HCPs should be prescribing naloxone, most HCP respondents selected pharmacists $(76.4 \%)$ and substance abuse providers $(71.6 \%)$. Less than half of respondents $(45.0 \%)$ felt that VA ED/UCC providers also should be prescribing naloxone. However, $58.1 \%$ of most HCP respondents reported being comfortable or very comfortable with prescribing naloxone to a patient in the ED or UCC who already had an existing prescription of opioids. Similarly, $52.7 \%$ of respondents reported being comfortable or very comfortable with coprescribing naloxone when discharging a patient with an opioid prescription from the ED/UCC. Notably, while $36.7 \%$ of PAs reported being comfortable/very comfortable coprescribing naloxone, $46.7 \%$ reported being comfortable/very comfortable prescribing naloxone to a patient with an existing opioid prescription. Physicians and NPs expressed similar levels of comfort with coprescribing and prescribing naloxone.

Respondents across provider types indicated a number of barriers to prescribing naloxone to medically appropriate patients (Table 2). Many respondents indicated prescribing naloxone was beyond the ED/UCC provider scope of practice (35.2\%), followed by the perceived stigma associated with naloxone (33.3\%), time required to prescribe naloxone $(23.9 \%)$, and concern with patient's ability to use naloxone (22.8\%).

Facilitators for prescribing naloxone to medically appropriate patients identified by HCP respondents included pharmacist help and education (44.6\%), patient knowledge 
TABLE 4 Barriers to MAT Initiation From ED and UCC

\begin{tabular}{lcccc} 
& \multicolumn{3}{c}{ Health Care Providers, No. (\%) } \\
\cline { 2 - 5 } Barriers & Physicians & $\begin{array}{c}\text { Nurse } \\
\text { Practitioners }\end{array}$ & $\begin{array}{c}\text { Physician } \\
\text { Assistants }\end{array}$ & All \\
\hline Cost & $17(6.0)$ & $5(8.2)$ & $3(10.0)$ & $25(6.7)$ \\
\hline Facility leadership not supportive & $20(7.1)$ & $4(6.6)$ & $5(16.7)$ & $29(7.8)$ \\
\hline Other & $22(7.8)$ & $5(8.2)$ & $2(6.7)$ & $29(7.8)$ \\
\hline Veterans unwilling/unable to use it & $47(16.7)$ & $17(27.9)$ & $6(20.0)$ & $70(18.8)$ \\
\hline $\begin{array}{l}\text { Heroin/opioid withdrawal is not life threatening; can } \\
\text { be handled in other ways in the ED/UCC }\end{array}$ & $58(20.6)$ & $11(18.0)$ & $3(10.0)$ & $72(19.4)$ \\
\hline Uncomfortable using MAT & $83(29.5)$ & $13(21.3)$ & $9(30.0)$ & $105(28.2)$ \\
\hline Time it requires to prescribe MAT & $89(31.7)$ & $16(26.2)$ & $6(20.0)$ & $111(29.8)$ \\
\hline Unclear follow-up plan and system for referral of care & $149(53.0)$ & $24(39.3)$ & $14(46.7)$ & $187(50.3)$ \\
\hline Beyond the ED/UCC provider scope of practice & $157(55.9)$ & $24(39.3)$ & $17(56.7)$ & $198(53.2)$
\end{tabular}

Abbreviations: CPRS, Computerized Patient Record System; ED, emergency department; MAT, medication-assisted treatment; UCC, urgent care center; VA, US Department of Veterans Affairs.

of medication options $(31.7 \%)$, societal shift away from opioids for pain management (28.0\%), facility leadership (26.9\%), and patient interest in safe opioid usage (26.6\%) (Table 3). In addition, NPs specifically endorsed Computerized Patient Record System prompts for patients prescribed opioids (29.5\%).

Less than $6.8 \%$ of HCP respondents indicated that they were comfortable using MAT. Meanwhile, $42.1 \%$ of respondents reported being aware of MAT but not familiar with it, and $23.5 \%$ reported that they were unaware of MAT. Correspondingly, 301 of the $372(88.5 \%)$ HCP respondents indicated that they had not prescribed MAT in the past year. Across HCP types, only $24.1 \%$ indicated that it is the role of VA ED or UCC providers to prescribe MAT when medically appropriate and subsequently refer patients to substance abuse treatment for follow-up (just $7.1 \%$ of PAs endorsed this). Furthermore, $6.5 \%$ and $18.8 \%$ of HCP respondents indicated that their facility leadership was very supportive and supportive, respectively, of MAT for OUD prescribing.

Barriers to MAT initiation indicated by HCP respondents included limited scope of ED and UCC practice (53.2\%), unclear follow-up/referral process $(50.3 \%)$, time (29.8\%), and discomfort (28.2\%). Nearly one-third of NPs (27.9\%) identified patient willingness/ability as a barrier to MAT initiation (Table 4).

Facilitators of MAT initiation in the ED or UCC included VHA same-day treatment options $(34.9 \%)$, patient desire $(32.5 \%)$, pharmacist help/education (27.4\%), and psychiatric social workers in the ED or UCC (25.3\%). Some NPs (23.0\%) and PAs $(26.7 \%)$ also indicated that having time to educate veterans about the medication would be a facilitator (Table 5). Facility leadership support was considered a facilitator by $30 \%$ of PAs.

\section{DISCUSSION}

To the best of our knowledge, there have not been any studies examining HCP perceptions of the barriers and facilitators to naloxone distribution or the initiation of MAT in VHA ED and UCCs. Veterans are at an increased risk of overdose when compared with the general population, and increasing access to opioid safety measures (eg, safer prescribing practices, naloxone distribution) and treatment with MAT for OUD across all clinical settings has been a VHA priority. ${ }^{3}$ 
TABLE 5 Facilitators to MAT Initiation from EDs and UCCs by HCP

\begin{tabular}{|c|c|c|c|c|}
\hline \multirow[b]{2}{*}{ Facilitators } & \multicolumn{4}{|c|}{ Health Care Providers, No. (\%) } \\
\hline & Physicians & $\begin{array}{c}\text { Nurse } \\
\text { Practitioners }\end{array}$ & $\begin{array}{l}\text { Physician } \\
\text { Assistants }\end{array}$ & All \\
\hline Veterans perceived stigma about MAT & $12(4.3)$ & $6(9.8)$ & $4(13.3)$ & $22(5.9)$ \\
\hline Pharmacist prompt in CPRS & $29(10.3)$ & $6(9.8)$ & $6(20.0)$ & $41(11.0)$ \\
\hline $\begin{array}{l}\text { Videos and/or handouts for patients with } \\
\text { education about medication }\end{array}$ & $35(12.5)$ & $9(14.8)$ & $5(16.7)$ & $49(13.2)$ \\
\hline Other & $45(16.0)$ & $10(16.4)$ & $3(10.0)$ & $58(15.6)$ \\
\hline $\begin{array}{l}\text { Being able to spend more time with the veteran } \\
\text { to educate him/her about the medication }\end{array}$ & $44(15.7)$ & $14(23.0)$ & $8(26.7)$ & $66(17.7)$ \\
\hline Facility leadership interest & $53(18.9)$ & $9(14.8)$ & $9(30.0)$ & $71(19.1)$ \\
\hline Patient knowledge of medication & $53(18.9)$ & $11(18.0)$ & $8(26.7)$ & $72(19.4)$ \\
\hline Psychiatric social workers in the ED/UCC & $73(26.0)$ & $12(19.7)$ & $9(30.0)$ & $94(25.3)$ \\
\hline $\begin{array}{l}\text { Pharmacist who could help prescribe and } \\
\text { educate about medication }\end{array}$ & $78(27.8)$ & $15(24.6)$ & $9(30.0)$ & $102(27.4)$ \\
\hline Patient desire to quit or taper from using opioids & $93(33.1)$ & $17(27.9)$ & $11(36.7)$ & $121(32.5)$ \\
\hline $\begin{array}{l}\text { Additional VA-based treatment options for the } \\
\text { same-day of presentation to the ED/UCC (eg, } \\
\text { detox facility) }\end{array}$ & $101(35.9)$ & $18(29.5)$ & $11(36.7)$ & $130(34.9)$ \\
\hline
\end{tabular}

National guidance from VHA leadership, the Centers for Disease Control and Prevention (CDC), the US Surgeon General, and the US Department of Health and Human Services (HHS) call for an all-hands-on-deck approach to combatting opioid overdose with naloxone distribution or MAT (such as buprenorphine) initiation. ${ }^{13}$ VHA ED and UCC settings provide acute outpatient care to patients with medical or psychiatric illnesses or injuries that the patient believes requires emergent or immediate medical attention or for which there is a critical need for treatment to prevent deterioration of the condition or the possible impairment of recovery. ${ }^{14}$ However, ED and UCC environments are often regarded as settings meant to stabilize a patient until they can be seen by a primary care or long-term care provider.

A major barrier identified by HCPs was that MAT for OUD was outside their ED/ UCC scope of practice, which suggests a need for a top-down or peer-to-peer reexamination of the role of HCPs in ED/UCC settings. Any naloxone distribution and/or MAT-initiation program in VHA ED/UCCs should consider education about the role of ED/UCC HCPs in opioid safety and treatment. According to a VHA Support Service Center (VSSC) employee report database, in fiscal year 2018, per diem/fee-basis and contract HCPs comprised nearly $40 \%$ of clinical emergency medicine physician fulltime equivalent employees, which presents a unique barrier to HCP education. Feebasis and per diem HCPs may be less aware of, engaged in, or committed to VHA goals. Additionally, short-term HCPs may have fewer opportunities for training and education regarding naloxone or MAT use.

Only $25.3 \%$ of HCPs reported that their facility leadership was supportive or very supportive of MAT prescribing. This suggests that facility leadership should be engaged in any efforts to implement a MATinitiation program in the facility's ED. Engaging leadership in efforts to implement ED-based MAT programs will allow for a 
better understanding of leadership goals as related to opioid safety and an opportunity to address concerns regarding prescribing MAT in the ED. We recommend engaging facility leadership early in MAT implementation efforts. Respectively, $12.4 \%$ and $28.2 \%$ of HCP respondents reported discomfort prescribing naloxone or using MAT, suggesting a need for more education. Similarly, only $6.8 \%$ of HCPs reported comfort with using MAT.

A consideration for implementing ED/ UCC-based MAT should be the inclusion of a training component. An evidence-based clinical treatment pathway that is appropriate to the ED/UCC setting and facility on the administration of MAT also could be beneficial. A clinical treatment pathway that includes ED/UCC-initiated discharge recommendations would address HCP concerns of unclear follow-up plans and system for referral of care. To this end, a key implementation task is coordinating with other outpatient services (eg, pain management clinic, substance use disorder treatment clinic) equipped for long-term patient follow-up to develop a system for referral of care. For example, as part of the clinical treatment pathway, an ED can develop a system of referral for patients initiated on MAT in the ED in which patients are referred for follow-up at the facility's substance use disorder treatment clinic to be seen within 72 hours to continue the administration of MAT (such as buprenorphine).

In addition to HCP education, results suggest that patient/veteran education regarding naloxone and/or MAT should be considered. HCPs indicated that having help from a pharmacist to educate the patient about the medications would be a facilitator to naloxone distribution and MAT initiation. Similarly, patient knowledge of the medications also was endorsed as a facilitator. As such, a consideration for any future ED/UCC-based naloxone distribution or MAT-initiation programs in the VHA should be patient education whether by a clinically trained professional or an educational campaign for veterans.

Expanded naloxone distribution and initiation of MAT for OUD for EDs/UCCs across the VHA could impact the lives of veterans on long-term opioid therapy, with
OUD, or who are otherwise at risk for opioid overdose. Steps taken to address the barriers and leverage the facilitators identified by HCP respondents can greatly reduce current obstacles to widespread implementation of ED/UCC-based naloxone distribution and MAT initiation nationally within the VHA.

\section{Limitations}

This survey had a low response rate (16.7\%). One potential explanation for the low response rate is that when the survey was deployed, many of the VHA ED/UCC physicians were per-diem employees. Per-diem physicians may be less engaged and aware of site facilitators or barriers to naloxone and MAT prescribing. This, too, may have potentially skewed the collected data. However, the survey did not ask HCPs to disclose their employment status; thus, exact rates of per diem respondents are unknown.

We aimed to capture only self-perceived barriers to prescribing naloxone and MAT in the ED, but we did not capture or measure HCP respondent's actual prescribing rates of MAT or naloxone. Understanding HCP perceptions of naloxone distribution and MAT initiation in the ED may have been further informed by comparing HCP responses to their actual clinical practice as related to their prescribing of these medications. In future research, we will link HCPs with the actual numbers of naloxone and MAT medications prescribed. Additionally, we do not know how many of these barriers or proposed facilitators will impact clinical practice.

\section{CONCLUSIONS}

A key aim for VHA leadership is to increase veteran access to naloxone distribution and MAT for OUD across clinical areas. The present study aimed to identify HCP perceptions of barriers and facilitators to the naloxone distribution and MAT-initiation programs in VHA ED/UCCs to inform the development of a targeted QI program to implement these opioid safety measures. Although the survey yielded a low response rate, results allowed us to identify important action items for our QI program, such as the development of clear protocols, follow-up plans, and systems for referral of care and HCP educational materials related to MAT and naloxone. We hope 
this work will serve as the basis for ED/UCCtailored programs that can provide customized educational programs for HCPs designed to overcome known barriers to naloxone and MAT initiation.

\section{Acknowledgments}

This work was supported by the VA Office of Specialty Care Services 10P11 and through funding provided by the Comprehensive Addiction and Recovery Act (CARA).

\section{Author affiliations}

Nathalie Dieujuste is a Research and Project Coordinator, Rachel Johnson-Koenke is a Social Work Researcher, and Comilla Sasson is an Emergency Medicine Physician and Health Service Researcher, all at the Rocky Mountain Regional Veterans Affairs (VA) Medical Center in Aurora, Colorado. Manuel Celedon is an Assistant Chief of Emergency Medicine, and Zahir Basrai is an Emergency Medicine Physician, both at the Greater Los Angeles VA Health Care System in California. Melissa Christopher is an Associate Chief Consultant for VA Pharmacy Benefits Management and the National Director for VA PBM Academic Detailing Service in San Diego, California. Jason Smith is a Pharmacy Benefit Manager for VISN 19 Academic Detailing Service in Denver, Colorado. Rachel Johnson-Koenke is an Assistant Professor at the University of Colorado College of Nursing in Aurora.

\section{Author disclosures}

The authors report no actual or potential conflicts of interest with regard to this article.

\section{Disclaimer}

The opinions expressed herein are those of the authors and do not necessarily reflect those of Federal Practitioner, Frontline Medical Communications Inc., the US Government, or any of its agencies. This article may discuss unlabeled or investigational use of certain drugs. Please review the complete prescribing information for specific drugs or drug combinations-including indications, contraindications, warnings, and adverse effects-before administering pharmacologic therapy to patients.

\section{References}

1. Substance Abuse and Mental Health Services Administration. Key substance use and mental health indicators in the united states: results from the 2018 National Survey on Drug Use and Health. Published August 2019. Accessed August 20, 2021. https://www.samhsa.gov/data/sites /default/files/cbhsq-reports/NSDUHNationalFindings Report2018/NSDUHNationalFindingsReport2018.pdf

2. Bohnert AS, Ilgen MA, Galea S, McCarthy JF, Blow FC. Accidental poisoning mortality among patients in the Department of Veterans Affairs Health System. Med Care. 2011;49(4):393-396. doi:10.1097/MLR.0b013e318202aa27

3. US Department of Veterans Affairs, Pharmacy Benefits Management Service. Recommendations for issuing naloxone rescue for the VA opioid overdose education and naloxone distribution (OEND) program. Published August 2016. Accessed August 20, 2021. https://www.pbm .va.gov/PBM/clinicalguidance/clinicalrecommendations /Naloxone_HCl_Rescue_Kits_Recommendations_for_Use .pdf

4. US Department of Defense, US Department of Veterans Affairs, Opioid Therapy for Chronic Pain Work Group. VA/DoD clinical practice guideline for opioid therapy for chronic pain. Published February 2017. Accessed August 20, 2021. https://www.va.gov/HOMELESS/nchav /resources/docs/mental-health/substance-abuse/VA _DOD-CLINICAL-PRACTICE-GUIDELINE-FOR-OPIOID -THERAPY-FOR-CHRONIC-PAIN-508.pdf

5. Barnett ML, Olenski $A R$, Jena $A B$. Opioid-prescribing patterns of emergency physicians and risk of longterm use. N Engl J Med. 2017;376(7):663-673. doi:10.1056/NEJMsa1610524

6. Shah A, Hayes CJ, Martin BC. Characteristics of initial prescription episodes and likelihood of long-term opioid use - United States, 2006-2015. MMWR Morb Mortal Wkly Rep. 2017;66(10):265-269. Published 2017 Mar 17. doi:10.15585/mmwr.mm6610a1

7. Clark AK, Wilder CM, Winstanley EL. A systematic review of community opioid overdose prevention and naloxone distribution programs. J Addict Med. 2014;8(3):153-163. doi:10.1097/ADM.0000000000000034

8. Coffin PO, Behar E, Rowe C, et al. Nonrandomized intervention study of naloxone coprescription for primary care patients receiving long-term opioid therapy for Pain. Ann Intern Med. 2016;165(4):245-252. doi:10.7326/M15-2771

9. Ma J, Bao YP, Wang RJ, et al. Effects of medicationassisted treatment on mortality among opioids users: a systematic review and meta-analysis. Mol Psychiatry. 2019;24(12):1868-1883. doi:10.1038/s41380-018-0094-5

10. D'Onofrio G, O'Connor PG, Pantalon MV, et al. Emergency department-initiated buprenorphine/naloxone treatment for opioid dependence: a randomized clinical trial. JAMA. 2015;313(16):1636-1644. doi:10.1001/jama.2015.3474

11. Dieujuste N, Johnson-Koenke R, Christopher M, et al. Feasibility study of a quasi-experimental regional opioid safety prescribing program in Veterans Health Administration emergency departments. Acad Emerg Med. 2020;27(8):734-741. doi:10.1111/acem.13980

12. Mackey K, Veazie S, Anderson J, Bourne D, Peterson K. Evidence brief: barriers and facilitators to use of medications for opioid use disorder. Published July 2017. Accessed August 20, 2021. http://www.ncbi.nlm.nih.gov /books/NBK549203/

13. US Department of Health and Human Services, Office of the Surgeon General. Naloxone: the opioid reversal drug that saves lives. Published December 2018. Accessed August 20, 2021. https://www.hhs.gov/opioids/sites/default /files/2018-12/naloxone-coprescribing-guidance.pdf

14. US Department of Veterans Affairs, Veterans Health Administration. Chapter 256: Emergency department (ED) and urgent care clinic (UCC). Updated October 3, 2016. Accessed August 20, 2021. https://www.cfm.va.gov/til /space/spChapter256.pdf. 